\title{
Hypernuclear structure with the Nijmegen ESC08 potentials
}

\author{
H.-J. Schulze ${ }^{1,2}$ and T. Rijken ${ }^{2,3}$ \\ ${ }^{1}$ INFN Sezione di Catania, Dipartimento di Fisica, Via Santa Sofia 64, I-95123 Catania, Italy \\ ${ }^{2}$ Yukawa Institute for Theoretical Physics, Kyoto University, Kyoto 606-8502, Japan \\ ${ }^{3}$ IMAPP, University of Nijmegen, 6525 AJ Nijmegen, The Netherlands
}

(Received 24 June 2013; published 23 August 2013)

\begin{abstract}
We perform Brueckner-Hartree-Fock calculations of hypernuclear matter employing the recent Nijmegen extended soft-core 08 hyperon-nucleon and hyperon-hyperon potentials. The results are used in a generalized Skyrme-Hartree-Fock model to calculate the properties of single- and double- $\Lambda$ hypernuclei. Based on those results, estimates of the effects of hypernuclear three-body forces and other corrections are made.
\end{abstract}

DOI: 10.1103/PhysRevC.88.024322

PACS number(s): 21.80.+a, 13.75.Ev, 21.60.Jz, 21.65.-f

\section{INTRODUCTION}

New and upgraded experimental facilities (DAFNE, FAIR, JLab, J-PARC, ...) now offer the possibility to determine the properties of single- and double- $\Lambda$ hypernuclei better than ever before [1]. This also challenges the development and improvement of theoretical approaches for hypernuclear structure and of (nonrelativistic potential) models [2-8] for the underlying bare nucleon-hyperon $(N Y)$ and hyperon-hyperon $(Y Y)$ interactions.

We examine in this article the predictions of the recently completed Nijmegen extended soft-core 08 (ESC08) $N Y$ and $Y Y$ potentials [8], which for the first time are based on a unified theoretical framework involving also the nucleon-nucleon $(N N)$ sector, minimizing the number of free parameters. Apart from features regarding the $\Lambda$ hyperon, of particular interest are also the properties of the $\Sigma$ and $(S=-2) \Xi$ hyperons, for which better experimental constraints are expected to become available soon, too.

Our theoretical method for this purpose is a two-step process: First, a reliable $N Y+Y Y$ in-medium interaction $(G$ matrix) is computed within a Brueckner-Hartree-Fock (BHF) approach of hypernuclear bulk matter $[9,10]$. This in-medium interaction is then used in a local-density approximation within a generalized Skyrme-Hartree-Fock (SHF) model for finite hypernuclei [11-13].

We first briefly review the ESC08 potentials in Sec. II, the BHF calculations of hypernuclear bulk matter in Sec. III, and the extended SHF approach for hypernuclei in Sec. IV. We then present the theoretical results for single- and double- $\Lambda$ hypernuclei in Sec. V, and estimate in Sec. VI the required corrections to our formalism by fitting experimental data within the same framework.

\section{THE NIJMEGEN ESC08 POTENTIAL}

The ESC08 model [8] for baryon-baryon interactions of the $\mathrm{SU}(3)$ flavor octet of baryons $(N, \Lambda, \Sigma$, and $\Xi$ ) provides a presentation of the forces in terms of (i) meson exchange, using generalized soft-core Yukawa functions; (ii) multiple gluon exchange (pomeron and odderon); and (iii) structural effects due to the quark core of the baryons, the so-called Pauli-blocking. Relativistic effects are included via expansions in inverse baryon masses $1 / m_{B}$. The ESC meson-exchange interactions contain local and nonlocal potentials due to (a) one boson exchanges (OBE), which are members of nonets of pseudoscalar, vector, scalar, and axial mesons; (b) pomeron and odderon exchanges; (c) two pseudoscalar exchanges (TME); and (d) meson pair exchanges (MPE). The OBE and MPE vertices are regulated by gaussian form factors, where the assignment of the cut-off masses for the baryon-baryonmeson (BBM) vertices depends on the SU(3) classification of the exchanged mesons for OBE, and a similar scheme for MPE.

The ESC models describe the $N N, N Y$, and $Y Y$ interactions in a unified way using broken flavor $\mathrm{SU}(3)$ symmetry. This serves to connect the $N N, N Y$, and $Y Y$ channels and is utilized to make a simultaneous fit to the $N N$ and $N Y$ data with a restricted set $(\lesssim 20)$ of free coupling constants, etc., see [8] for details. In particular, the BBM coupling constants are calculated via $\mathrm{SU}(3)$, using, together with the meson mixing angles, the fitted constants in the $N N \oplus N Y$ analysis as input. In ESC08 no breaking of SU(3) is assumed for the couplings with the exception of the following cases: (i) $N N$ : the isospin breaking for the $\rho$ meson is exploited phenomenologically in order to account for the difference between ${ }^{1} S_{0}(p p),{ }^{1} S_{0}(n p)$, and ${ }^{1} S_{0}(n n)$; (ii) Charge symmetry breaking in the $\Lambda p$ and $\Lambda n$ channels, where we include the $\mathrm{SU}(2)$ isospin breaking in the OBE, TME, and MPE potentials.

In this paper solution ESC08b [8] is used as a basis for the $N Y$ interactions. This model achieves, with single sets of parameters and without ad hoc changes of the rules in particular channels, excellent results for the $N N$ and $N Y$ data: (i) For the selected $4233 N N$ data of the Nijmegen phase shift analysis [14] with energies $0 \leqslant T_{\text {lab }} \leqslant 350 \mathrm{MeV}$ a $\chi^{2} /$ data $=1.157$ is realized, which is close to that of the multienergy phase shift analysis [14]. (ii) For the set of $38 N Y$ $S=-1$ data, also used in previous Nijmegen studies, in $\mathrm{ESC} 08 \mathrm{~b} \chi^{2} /$ data $\approx 0.65$ was reached, without bound states in these $N Y$ channels. As regards the $U_{\Lambda}$ well-depth there is some overbinding, making room for, e.g., three-body repulsion. (iii) For $Y Y$ there is a weak $\Lambda \Lambda$ attraction, e.g., in ESC08 $\left|a_{\Lambda \Lambda}\left({ }^{1} S_{0}\right)\right|<1.0$, which matches experimental indication from the Nagara event [15]. Among the predictions for the $S=-2$ sector $(N \Xi, \Lambda \Lambda, \Lambda \Sigma, \Sigma \Sigma)$ are the existence of bound states in the $N \Xi\left({ }^{3} S_{1}-{ }^{3} D_{1}, T=1\right)$ channel. 
TABLE I. Meson coupling constants (at $\boldsymbol{k}^{2}=0$ ) and parameters employed in the ESC08b OBE potentials. The masses and $\Lambda$ 's are given in $\mathrm{MeV}$.

\begin{tabular}{lrrrcc}
\hline \hline Meson & Mass & $g / \sqrt{4 \pi}$ & $f / \sqrt{4 \pi}$ & $\Lambda$ & SU(3) \\
\hline$\pi$ & 138.04 & & 0.2652 & 872.09 & $\alpha_{P}=0.4281$ \\
$\eta$ & 547.45 & & 0.1655 & 893.74 & $\theta_{P}=-23.00^{\circ}$ \\
$\eta^{\prime}$ & 957.75 & & 0.1104 & $\#$ & \\
$\rho$ & 768.10 & 0.7783 & 3.9335 & 707.62 & $\alpha_{V}^{e}=1.0000$ \\
$\phi$ & 1019.41 & -0.9043 & 3.7689 & $”$ & $\alpha_{V}^{m}=0.5411$ \\
$\omega$ & 781.95 & 3.3928 & -0.5681 & 1116.48 & $\theta_{V}=37.50^{\circ}$ \\
$a_{1}$ & 1270.00 & -1.1875 & 0.1888 & 1254.79 & $\alpha_{A}=0.0211$ \\
$f_{1}$ & 1420.00 & 0.0180 & -0.2423 & $”$ & $\theta_{A}=-40.50^{\circ}$ \\
$f_{1}^{\prime}$ & 1285.00 & -0.9455 & -0.1300 & $”$ & \\
$b_{1}$ & 1235.00 & & -0.0404 & 872.09 & $\alpha_{B}=0.4281$ \\
$h_{1}$ & 1380.00 & & -0.0378 & 893.74 & $\theta_{B}=-23.00^{\circ}$ \\
$h_{1}^{\prime}$ & 1170.00 & & -0.0464 & $”$ & \\
$a_{0}$ & 962.00 & 1.1018 & & 1193.39 & $\alpha_{S}=0.7514$ \\
$f_{0}$ & 993.00 & -1.5277 & &, & $\theta_{S}=37.50^{\circ}$ \\
$\varepsilon$ & 760.00 & 4.0890 & & 1146.09 & \\
Pomeron & 267.93 & 3.3347 & & & $a_{P B}=0.149$ \\
Odderon & 454.55 & 0.5390 & -1.1610 & & \\
\hline \hline
\end{tabular}

In Tables I and II the OBE and MPE couplings are given for the solution of the ESC08b model, which is the basis for the computations of the present paper. See Ref. [8] for the definition of the model parameters. The $\mathrm{SU}(3) \alpha=$ $F /(F+D)$ parameters and meson-mixing angles $\theta$ enable the calculation of all BBM-couplings, etc. The $a_{P B}$ parameter gives the fraction of the pomeron coupling that is related to the PB effect.

\section{BHF CALCULATION OF HYPERNUCLEAR MATTER}

Our results are based on generalized BHF calculations $[9,16]$ [employing continuous single-particle (s.p.) potentials in the computation of the $G$ matrices] of $\Lambda$ hypermatter, i.e., baryonic matter characterized by partial densities $\rho_{q} ; q=$ $p, n, \Lambda$. The basic input quantities in the Bethe-Goldstone equation are the $N N, N Y$, and $Y Y$ potentials. In the current work we use the Argonne $V_{18} N N$ potential [17] supplemented by the microscopic nucleonic three-body forces (TBF) of Ref. [18] in order to ensure good saturation properties of pure

TABLE II. Pair-meson coupling constants (at $\boldsymbol{k}^{2}=0$ ) employed in the ESC08b MPE potentials.

\begin{tabular}{ccccc}
\hline \hline$J^{P C}$ & SU(3)-irrep & $(\alpha \beta)$ & $g / 4 \pi$ & $F /(F+D)$ \\
\hline $0^{++}$ & $\{1\}$ & $g(\pi \pi)_{0}$ & - & - \\
$0^{++}$ & $\#$ & $g(\sigma \sigma)$ & - & - \\
$0^{++}$ & $\{8\}_{s}$ & $g(\pi \eta)$ & -0.0210 & 1.000 \\
$1^{--}$ & $\{8\}_{a}$ & $g(\pi \pi)_{1}$ & 0.0012 & 1.000 \\
$1^{--}$ & $"$ & $f(\pi \pi)_{1}$ & -0.2345 & 0.400 \\
$1^{++}$ & $"$ & $g(\pi \rho)_{1}$ & 0.6197 & -0.278 \\
$1^{++}$ & $"$ & $g(\pi \sigma)$ & -0.0298 & -0.278 \\
$1^{++}$ & $"$ & $g(\pi P)$ & - & - \\
$1^{+-}$ & $\{8\}_{s}$ & $g(\pi \omega)$ & -0.0689 & 0.428 \\
\hline \hline
\end{tabular}

nuclear matter, and the ESC08 [8] $N Y$ and $Y Y$ potentials. For comparison some results obtained before [11] with the older NSC89 [3] and NSC97 [7] potentials will also be shown. We recall that the NSC89 potential contains no $Y Y$ components, whereas the ESC08 model comprises the full set of interactions in the strangeness $S=-1$ and $S=-2$ channels, namely in the isospin basis it treats the coupled states

$$
\begin{array}{ll}
S=-1: & T=1 / 2: \quad N \Sigma, N \Lambda, \\
& T=3 / 2: \quad N \Sigma, \\
S=-2: \quad & T=0: \quad \Sigma \Sigma, N \Xi, \Lambda \Lambda, \\
& T=1: \quad \Sigma \Sigma, N \Xi, \Lambda \Sigma, \\
& T=2: \quad \Sigma \Sigma .
\end{array}
$$

Using these potentials we have to solve the BetheGoldstone integral equation $[9,19]$ for the correlated wave functions $u$ in the various $N N, N Y$, and $Y Y$ channels:

$$
\begin{aligned}
u_{C C^{\prime}, L L^{\prime}}(k, r)= & j_{L}(k r) \delta_{C C^{\prime}} \delta_{L L^{\prime}} \\
& +\frac{2}{\pi} \int_{0}^{\infty} d r^{\prime} r^{\prime 2} D_{C C^{\prime}, L^{\prime}}\left(k, r, r^{\prime}\right) \\
& \times \sum_{C^{\prime \prime}, L^{\prime \prime}} V_{C^{\prime} C^{\prime \prime}, L^{\prime} L^{\prime \prime}}\left(r^{\prime}\right) u_{C C^{\prime \prime}, L L^{\prime \prime}}\left(k, r^{\prime}\right)
\end{aligned}
$$

with the intermediate propagator

$$
D_{C C^{\prime}, L^{\prime}}\left(k, r, r^{\prime}\right)=\int_{0}^{\infty} d k^{\prime} k^{\prime 2} \frac{j_{L^{\prime}}\left(k^{\prime} r\right) j_{L^{\prime}}\left(k^{\prime} r^{\prime}\right) f_{C^{\prime}}\left(k^{\prime}\right)}{E_{C}(k)-E_{C^{\prime}}\left(k^{\prime}\right)+i \varepsilon}
$$

and

$E_{C}(k)=e_{c_{1}}\left(k_{1}\right)+e_{c_{2}}\left(k_{2}\right), \quad e_{c}(k)=\frac{k^{2}}{2 M_{c}}+\operatorname{Re} U_{c}(k)+M_{c}$.

Here $C=c_{1} c_{2}, C^{\prime}, C^{\prime \prime}$ denote channel indices representing baryon pairs, $k$ and $k^{\prime}$ denote the relative momenta of the initial $C$ and the intermediate $C^{\prime}$ state, $E_{C}(k)$ and $E_{C^{\prime}}\left(k^{\prime}\right)$ are the corresponding pair energies, and $f_{C^{\prime}}\left(k^{\prime}\right)$ is the angleaveraged Pauli operator in the intermediate states. More details on these quantities are given in Ref. [9]. The equation has to be solved for a set of states with definite quantum numbers $T, S, J$, which have not been indicated explicitly. In practice we consider all partial waves up to $L=7$ in the $N N$ and $L=$ 5 in the $N Y$ and $Y Y$ sectors. The Bethe-Goldstone equation has thus a $1 \times 1,2 \times 2$, or $3 \times 3$ matrix structure according to the relevant isospin channel, Eqs. (1)-(5); for example in schematic notation (now 1,2,3 denote pairs of baryons)

$$
\left(\begin{array}{l}
u_{11} \\
u_{12} \\
u_{13}
\end{array}\right)=\left(\begin{array}{c}
j_{L} \\
0 \\
0
\end{array}\right)+\left(\begin{array}{lll}
D_{11} V_{11} & D_{11} V_{12} & D_{11} V_{13} \\
D_{12} V_{21} & D_{12} V_{22} & D_{12} V_{23} \\
D_{13} V_{31} & D_{13} V_{32} & D_{13} V_{33}
\end{array}\right)\left(\begin{array}{l}
u_{11} \\
u_{12} \\
u_{13}
\end{array}\right),
$$

with possible permutations, and an additional $2 \times 2$ structure when the mixing of angular momentum states through the tensor potential applies.

The solutions of the Bethe-Goldstone equation determine the diagonal $G$-matrix elements

$$
\begin{aligned}
& \left\langle k_{1} k_{2}\left|G_{C, L}^{T S J}\right| k_{1} k_{2}\right\rangle \\
& \quad=4 \pi \int_{0}^{\infty} d r r^{2} j_{L}(k r) \sum_{C^{\prime}, L^{\prime}} V_{C C^{\prime}, L L^{\prime}}(r) u_{C C^{\prime}, L L^{\prime}}(k, r),
\end{aligned}
$$


and the s.p. potentials (in the so-called continuous choice) are then given by

$$
\begin{aligned}
U_{a}^{(b)}\left(k_{a}\right)= & \sum_{T, S, J, L} \frac{(2 T+1)(2 J+1)}{\left(2 t_{a}+1\right)\left(2 s_{a}+1\right)} \\
& \times \int^{k_{F}^{(b)}} \frac{d^{3} k_{b}}{(2 \pi)^{3}}\left\langle k_{a} k_{b}\left|G_{a b, L}^{T S J}\right| k_{a} k_{b}\right\rangle,
\end{aligned}
$$

where the notation $U_{a}^{(b)}$ denotes the s.p. potential of particle $a$ due to the interaction with particles $b$ in the medium. Carrying out the calculation for the relevant combinations $a=N, \Lambda, \Sigma, \Xi ; b=N, \Lambda$, we obtain the total s.p. potentials of nucleons and hyperons as

$$
U_{a}(k)=U_{a}^{(N)}(k)+U_{a}^{(\Lambda)}(k) .
$$

Due to the occurrence of the $U_{a}$ in Eq. (8), the set of equations (6)-(12) constitutes a coupled system that has to be solved in a self-consistent manner.

We are interested in the total binding energy per baryon $B / A$, or equivalently the energy density $\varepsilon_{\mathrm{BHF}}$ of the bulk matter. In the BHF approximation this quantity and the baryon number $A$ per unit volume, i.e., the baryon density $\rho$, are given by

$$
\begin{aligned}
\varepsilon_{\mathrm{BHF}}=\frac{B}{A} \rho= & \frac{4 \pi}{(2 \pi)^{3}}\left[4 \int_{0}^{k_{F}^{(N)}} d k k^{2}\left(\frac{k^{2}}{2 M_{N}}+\frac{1}{2} U_{N}(k)\right)\right. \\
& \left.+2 \int_{0}^{k_{F}^{(\Lambda)}} d k k^{2}\left(\frac{k^{2}}{2 M_{\Lambda}}+\frac{1}{2} U_{\Lambda}(k)\right)\right]
\end{aligned}
$$

and

$$
\rho=\rho_{N}+\rho_{\Lambda}=\frac{1}{3 \pi^{2}}\left(2 k_{F}^{(N)^{3}}+k_{F}^{(\Lambda)^{3}}\right) .
$$

In the following we will make use of the principal results of the calculations, which are the energy density as function of the nucleon and $\Lambda$ partial densities, $\varepsilon_{\mathrm{BHF}}\left(\rho_{N}, \rho_{\Lambda}\right)$, as well as the momentum-dependent s.p. potentials of all types of particles involved, $U_{a}(k)$.

\section{A. Results}

In order to illustrate the basic features, Fig. 1 shows the complete set of nucleon and hyperon s.p. potentials in pure symmetric nuclear matter at saturation density $\rho_{N}=$ $\rho_{0}=0.17 \mathrm{fm}^{-3}$ (left panel) and in hypernuclear matter with densities $\rho_{N}=\rho_{0}, \rho_{\Lambda}=\rho_{0} / 2$ (right panel), resulting from our calculations with the ESC08 potential. One notes that the hyperon s.p. potentials are much less attractive than the nucleonic ones, reflecting the weaker strength of the $N Y$ compared to the $N N$ potentials. Within the ESC08 model, the well-depths $U(k=0)$ in normal nuclear matter of the $\Lambda, \Sigma$, and $\Xi$ hyperons are, respectively, $-39,+16,-8 \mathrm{MeV}$, and thus in reasonable agreement with current experimental estimates of these quantities $[20,21]$. The partial wave decompositions of these values are given in Table III which also lists explicitly the contributions of the different (isospin) channels according to Eqs. (10) and (11). One notes, in particular for the $\Sigma$ and $\Xi$ hyperons, strong cancellations between the individual partial

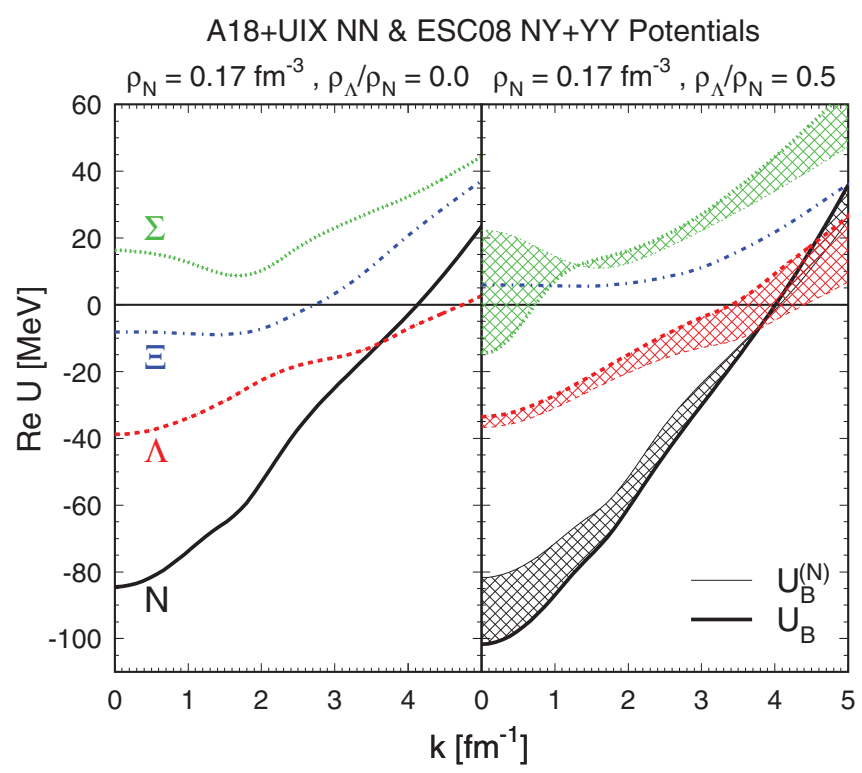

FIG. 1. (Color online) Nucleon and hyperon, $B=N, \Lambda, \Sigma, \Xi$, s.p. potentials (real parts) for $\rho_{N}=0.17 \mathrm{fm}^{-3}$ and $\rho_{\Lambda}=0$ (left panel) or $\rho_{\Lambda}=\rho_{N} / 2$ (right panel). The right panel displays $U_{B}$ (thick curves) and $U_{B}^{(N)}$ (thin curves). The hatched areas thus represent $U_{B}^{(\Lambda)}$.

wave contributions. For example, in the case of the $\Xi$, the most important contributions are the $N \Xi-N \Xi(T=1){ }^{3} S_{1}$ and the $N \Xi-\Sigma \Lambda(T=1){ }^{3} S D_{1}$ channels with magnitudes of about $50 \mathrm{MeV}$, but opposite sign, while the final $(T=1)$ result is of the order of $1 \mathrm{MeV}$. Clearly a sufficient numerical accuracy is required to handle this feature reliably, and it is also obvious that none of the coupled channels can be disregarded.

The effect of the presence of $\Lambda$ 's in nuclear matter can be seen in the right panel of Fig. 1: The attractive $N \Lambda$ interaction provides a deeper mean field for the nucleons (thick vs. thin solid black curves), whereas the $(T=0) \Lambda \Lambda-\Sigma \Sigma-N \Xi$ interaction has a small repulsive effect on the $\Lambda$ (dashed red curves), while the $(T=1) \Sigma \Lambda-\Sigma \Sigma-N \Xi$ channel generates substantial attraction for low-momentum $\Sigma$ 's (dotted green curves). The $\Xi \Lambda(S=-3)$ interaction is not considered in the present model and thus the $\Xi$ s.p. potential (dash-dotted blue curve) is only affected indirectly (via the modification of the various s.p. potentials) by the presence of $\Lambda$ 's, which yields a somewhat repulsive effect, as can be seen comparing the left and right panels of Fig. 1.

The most relevant features that can be extracted from plots like Fig. 1 are the $\Lambda$ effective mass, Eq. (18), and the "well depth" [22] $U_{\Lambda}^{0} \equiv \operatorname{Re} U_{\Lambda}(k=0)$. This quantity is slightly more attractive than the relevant SHF potential $V_{\Lambda}$, Eq. (23), due to rearrangement contributions to the latter [11]. The corresponding results are displayed in Fig. 2 (top and central panels) as a function of the nucleonic density for pure nuclear matter $\left(\rho_{\Lambda}=0\right)$. Comparing the new ESC08 results with the old NSC89 ones, one notes a slightly stronger effective $N \Lambda$ attraction, namely, the $\Lambda$ is more bound $\left(V_{\Lambda} \approx-36\right.$ vs. $\approx-28 \mathrm{MeV}$ at $\rho_{N}=\rho_{0}$ ) and its effective mass is lower $(0.74$ vs. 0.82$)$ with the former potential. This will have 
TABLE III. The contributions (in MeV) of various partial waves to the s.p. potentials $\operatorname{Re} U_{Y}(k=0)$ at $\rho_{N}=0.17 \mathrm{fm}^{-3}$ and $\rho_{\Lambda}=0$. Entries denoted " 0 " vanish in the isospin basis. The total sums include partial waves up to $L=5$.

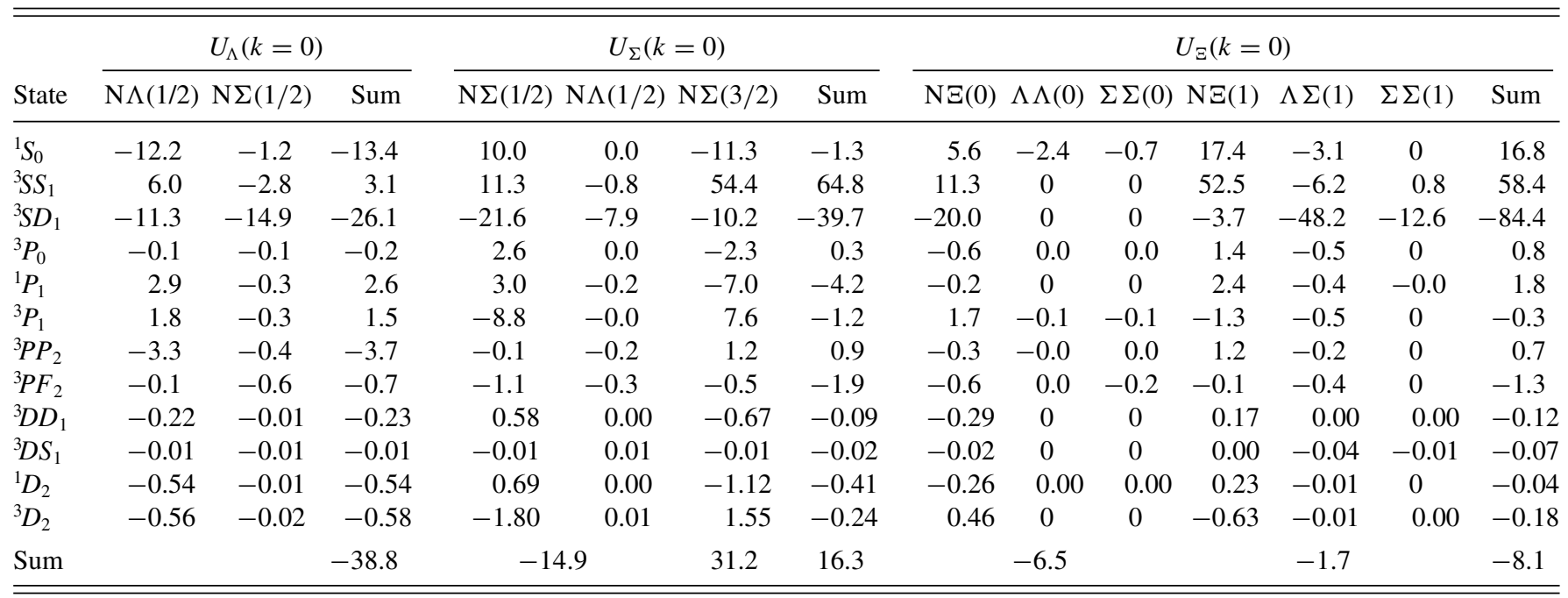

obvious effects on the predicted s.p. spectra of $\Lambda$-hypernuclei that we investigate now.

For later use we also plot in the lower panel of Fig. 2 the s.p. potential component $\operatorname{Re} U_{\Lambda}^{(\Lambda)}(k=0)$, Eqs. (11) and (12),

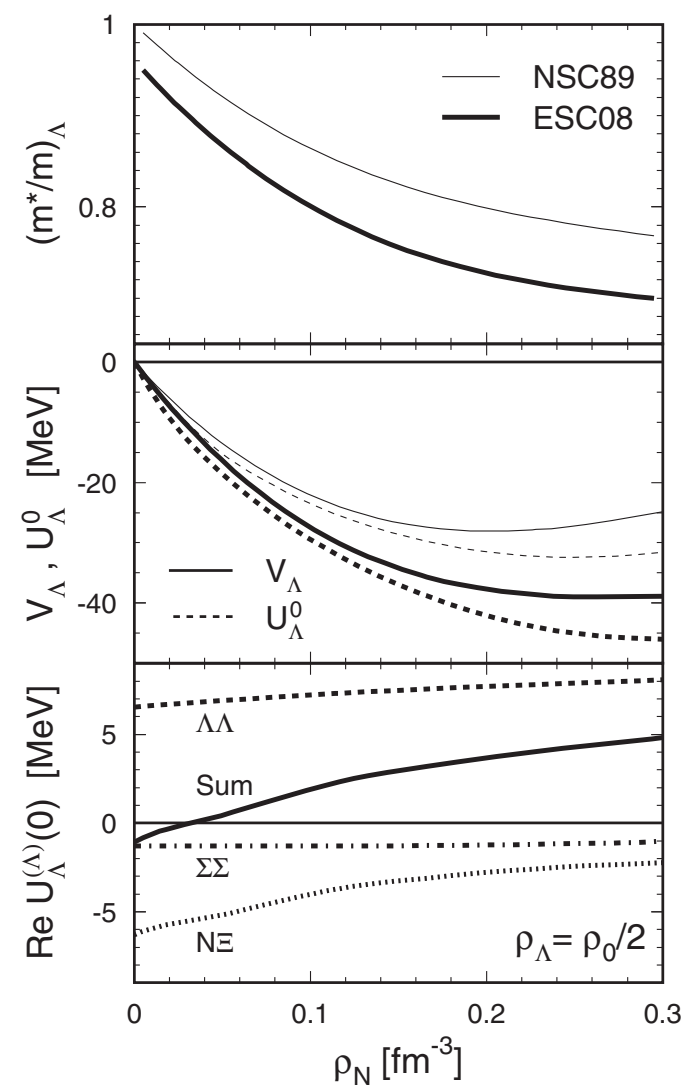

FIG. 2. $\Lambda$ effective mass (upper panel), $\Lambda$ well depth (central panel), and $\operatorname{Re} U_{\Lambda}^{(\Lambda)}(k=0)$ with different contributions (lower panel) in symmetric nuclear matter of density $\rho_{N}$, obtained with the ESC08 (thick curves) and NSC89 (thin curves, upper and central panels) potentials. of the ESC08 model in hypernuclear matter with varying $\rho_{N}$ and fixed $\rho_{\Lambda}=\rho_{0} / 2$, and its contributions from the different coupled channels $(T=0) \Lambda \Lambda, \Sigma \Sigma, N \Xi$. The total result (solid curve) changes from weak attraction $(-1.1 \mathrm{MeV})$ in free space to repulsion in nuclear matter $\left(+3.2 \mathrm{MeV}\right.$ at $\left.\rho_{N}=\rho_{0}\right)$ and is the result of strong compensation of the individual contributions, in particular $\Lambda \Lambda-\Lambda \Lambda$ (repulsive) and $\Lambda \Lambda-N \Xi$ (attractive). This balance changes with increasing nuclear density, and the overall effect turns from attraction to repulsion. The free $\Lambda \Lambda$ interaction can therefore not be used to estimate the in-medium behavior of two $\Lambda$ 's.

\section{SHF CALCULATION OF HYPERNUCLEI}

In our approach the local energy density functional of hypernuclear matter depends on the one-body densities $\rho_{q}$, kinetic densities $\tau_{q}$, and spin-orbit currents $\boldsymbol{J}_{q}$,

$\left[\rho_{q}, \tau_{q}, \boldsymbol{J}_{q}\right]=\sum_{i=1}^{N_{q}} n_{q}^{i}\left[\left|\phi_{q}^{i}\right|^{2},\left|\nabla \phi_{q}^{i}\right|^{2}, \phi_{q}^{i *}\left(\nabla \phi_{q}^{i} \times \sigma\right) / i\right]$,

where $\phi_{q}^{i}\left(i=1, N_{q}\right)$ are the s.p. wave functions of the $N_{q}$ occupied states for the species $q=n, p, \Lambda$ in a hypernucleus. The functional is written as $\varepsilon_{\mathrm{SHF}}=\varepsilon_{N}+\varepsilon_{\Lambda}$, where $\varepsilon_{N}$ is the standard purely nucleonic SHF functional [23,24], and

$$
\varepsilon_{\Lambda}=\frac{\tau_{\Lambda}}{2 m_{\Lambda}}+\varepsilon_{N \Lambda}\left(\rho_{N}, \rho_{\Lambda}\right)
$$

is the functional accounting for the presence of $\Lambda$ 's, due to the action of $N Y$ and $Y Y$ forces.

It can be constructed from the BHF energy density of bulk matter, Eq. (13), as

$$
\varepsilon_{N \Lambda}\left(\rho_{N}, \rho_{\Lambda}\right)=\varepsilon_{\mathrm{BHF}}\left(\rho_{N}, \rho_{\Lambda}\right)-\varepsilon_{\mathrm{BHF}}\left(\rho_{N}, 0\right)-\frac{C \rho_{\Lambda}^{5 / 3}}{2 m_{\Lambda}},
$$

where the last term corresponds to the kinetic energy contribution of the $\Lambda$ 's in bulk matter. The constant $C=$ $(3 / 5)\left(3 \pi^{2}\right)^{2 / 3} \approx 5.742$ has been introduced. However, we 
consider more realistic to work with a Schrödinger equation that involves, instead of the bare $\Lambda$ mass $m_{\Lambda}$, the hyperon effective mass $m_{\Lambda}^{*}$, as extracted from the BHF s.p. potential $U_{\Lambda}(k)$ (see Fig. 1),

$$
\frac{m_{\Lambda}^{*}}{m_{\Lambda}}=\left(1+\frac{U_{\Lambda}\left(k_{F}^{(\Lambda)}\right)-U_{\Lambda}(0)}{k_{F}^{(\Lambda)^{2}} / 2 m_{\Lambda}}\right)^{-1} .
$$

For this purpose the energy density functional is written instead as

$$
\varepsilon_{\Lambda}=\frac{\tau_{\Lambda}}{2 m_{\Lambda}^{*}\left(\rho_{N}, \rho_{\Lambda}\right)}+\tilde{\varepsilon}_{N \Lambda}\left(\rho_{N}, \rho_{\Lambda}\right)
$$

with

$$
\tilde{\varepsilon}_{N \Lambda}\left(\rho_{N}, \rho_{\Lambda}\right)=\varepsilon_{N \Lambda}\left(\rho_{N}, \rho_{\Lambda}\right)-\left(\frac{m_{\Lambda}}{m_{\Lambda}^{*}\left(\rho_{N}, \rho_{\Lambda}\right)}-1\right) \frac{C \rho_{\Lambda}^{5 / 3}}{2 m_{\Lambda}} .
$$

Minimizing the total energy of the hypernucleus, $E=$ $\int d^{3} r \varepsilon_{\mathrm{SHF}}(r)$, one arrives with Eq. (19) at the SHF Schrödinger equation

$$
\left[\nabla \cdot \frac{1}{2 m_{q}^{*}(\boldsymbol{r})} \nabla-V_{q}(\boldsymbol{r})+i \boldsymbol{W}_{q}(\boldsymbol{r}) \cdot(\nabla \times \boldsymbol{\sigma})\right] \phi_{q}^{i}(\boldsymbol{r})=e_{q}^{i} \phi_{q}^{i}(\boldsymbol{r})
$$

with the mean fields

$$
\begin{aligned}
& V_{N}=V_{N}^{\mathrm{SHF}}+\frac{\partial \tilde{\varepsilon}_{N \Lambda}}{\partial \rho_{N}}, \\
& V_{\Lambda}=\frac{\partial \tilde{\varepsilon}_{N \Lambda}}{\partial \rho_{\Lambda}},
\end{aligned}
$$

where $V_{N}^{\text {SHF }}$ is the nucleonic Skyrme mean field without hyperons and $\boldsymbol{W}_{N}$ the nucleonic spin-orbit mean field [23]. At the present level of approximation we do not include a spin-orbit force for the $\Lambda$, which is justified by the experimental observation of very small $(\lesssim 0.2 \mathrm{MeV}) N \Lambda$ spin-orbit splittings [25]. An approximate center of mass correction is applied as usual [23] by replacing the bare masses:

$$
\frac{1}{m_{q}} \rightarrow \frac{1}{m_{q}}-\frac{1}{M},
$$

where $M=\left(N_{n}+N_{p}\right) m_{N}+N_{\Lambda} m_{\Lambda}$ is the total mass of the hypernucleus.

Solving the equation provides the wave functions $\phi_{q}^{i}(\boldsymbol{r})$ and the s.p. energies $-e_{q}^{i}$ for the different s.p. levels $i$ and species $q$. We use in this work the standard nucleonic Skyrme force SLy4 [26], but the results for hyperonic observables hardly depend on that choice.

For an efficient numerical procedure we provide parametrizations of the numerical results for the key quantities $\varepsilon_{N \Lambda}$, Eq. (17), and $m_{\Lambda}^{*}$, Eq. (18), in the following functional forms $\left(\rho_{N}\right.$ and $\rho_{\Lambda}$ given in units of $\mathrm{fm}^{-3}, \varepsilon_{N \Lambda}$ in $\left.\mathrm{MeV} \mathrm{fm}^{-3}\right)$ :

$$
\begin{aligned}
\varepsilon_{N \Lambda}\left(\rho_{N}, \rho_{\Lambda}\right) \approx & -\left(\varepsilon_{1}-\varepsilon_{2} \rho_{N}+\varepsilon_{3} \rho_{N}^{2}\right) \rho_{N} \rho_{\Lambda} \\
& +\left(\varepsilon_{4}-\varepsilon_{5} \rho_{N}+\varepsilon_{6} \rho_{N}^{2}\right) \rho_{N} \rho_{\Lambda}^{5 / 3} \\
& -\left(\varepsilon_{7}-\varepsilon_{8} \rho_{\Lambda}+\varepsilon_{9} \rho_{\Lambda}^{2}\right) \rho_{\Lambda}^{2}, \\
\frac{m_{\Lambda}^{*}}{m_{\Lambda}}\left(\rho_{N}\right) \approx & \mu_{1}-\mu_{2} \rho_{N}+\mu_{3} \rho_{N}^{2}-\mu_{4} \rho_{N}^{3} .
\end{aligned}
$$

TABLE IV. Parameters of the functionals of energy density and $\Lambda$ effective mass, Eqs. (25), (26), and (29), for the different $N Y$ potentials.

\begin{tabular}{lcccc}
\hline \hline & NSC89 & NSC97a & NSC97f & ESC08 \\
\hline$\varepsilon_{1}$ & 327 & 423 & 384 & 390 \\
$\varepsilon_{2}$ & 1159 & 1899 & 1473 & 1269 \\
$\varepsilon_{3}$ & 1163 & 3795 & 1933 & 1356 \\
$\varepsilon_{4}$ & 335 & 577 & 635 & 470 \\
$\varepsilon_{5}$ & 1102 & 4017 & 1829 & 1472 \\
$\varepsilon_{6}$ & 1660 & 11061 & 4100 & 2293 \\
$\varepsilon_{7}$ & 0 & 38 & 50 & 20 \\
$\varepsilon_{8}$ & 0 & 186 & 545 & 298 \\
$\varepsilon_{9}$ & 0 & 22 & 981 & 383 \\
$\tilde{\varepsilon}_{1}$ & -116 & & & 204 \\
$\tilde{\varepsilon}_{2}$ & 745 & & & 1230 \\
$\tilde{\varepsilon}_{3}$ & 0 & & & 0 \\
$\mu_{1}$ & 1 & 0.98 & 0.93 & 0.95 \\
$\mu_{2}$ & 1.83 & 1.72 & 2.19 & 1.80 \\
$\mu_{3}$ & 5.33 & 3.18 & 3.89 & 3.24 \\
$\mu_{4}$ & 6.07 & 0 & 0 & 0 \\
\hline \hline
\end{tabular}

The parameters $\varepsilon_{i}$ and $\mu_{i}$ are listed in Table IV for the different $N Y$ potentials that we use. These parametrizations have been obtained from BHF calculations of symmetric nuclear matter and therefore depend only on the total nucleonic density $\rho_{N}$. This is a fairly good approximation for the isoscalar $\Lambda$ hyperon and the nearly symmetric nuclei that we will consider here.

The functional form of Eq. (26) is purely phenomenological, whereas that of Eq. (25) is guided by the fact that the energy density functional can be related to the BHF s.p. potentials in the following manner [11]:

$$
\begin{aligned}
\varepsilon_{N \Lambda}\left(\rho_{N}, \rho_{\Lambda}\right)= & \sum_{k<k_{F}^{(\Lambda)}}\left[2 U_{\Lambda}^{(N)}(k)+U_{\Lambda}^{(\Lambda)}(k)\right] \\
& +2 \sum_{k<k_{F}^{(N)}}\left[\left.U_{N}^{(N)}(k)\right|_{\rho_{\Lambda}}-\left.U_{N}^{(N)}(k)\right|_{\rho_{\Lambda}=0}\right],
\end{aligned}
$$

which follows directly from Eqs. (12), (13), and (17).

\section{RESULTS}

The most significant results are the $\Lambda$ s.p. energies $e_{\Lambda}^{i}(i=$ $1 s, 1 p, 1 d, 1 f, 1 g$ ) of various single- $\Lambda$ hypernuclei, for which experimental results are available $[27,28]$. Figure 3 (top panel) compares the values obtained with the different potentials. Consistent with the results displayed in Fig. 2, the ESC08 force provides more binding than the NSC89, such that heavy hypernuclei result overbound with the former and underbound with the latter potential. Nevertheless the discrepancies are not dramatic in view of the microscopic, parameter-free approach that we are following here. In the next section we will discuss this issue further.

Of particular interest for current and future hypernuclear experiments are the properties of double- $\Lambda$ hypernuclei, since they might provide access to features of the $\Lambda \Lambda$ interaction. Recent experimental results [15] point to a fairly weak (attractive) effective $\Lambda \Lambda$ force. The ESC08 potential 


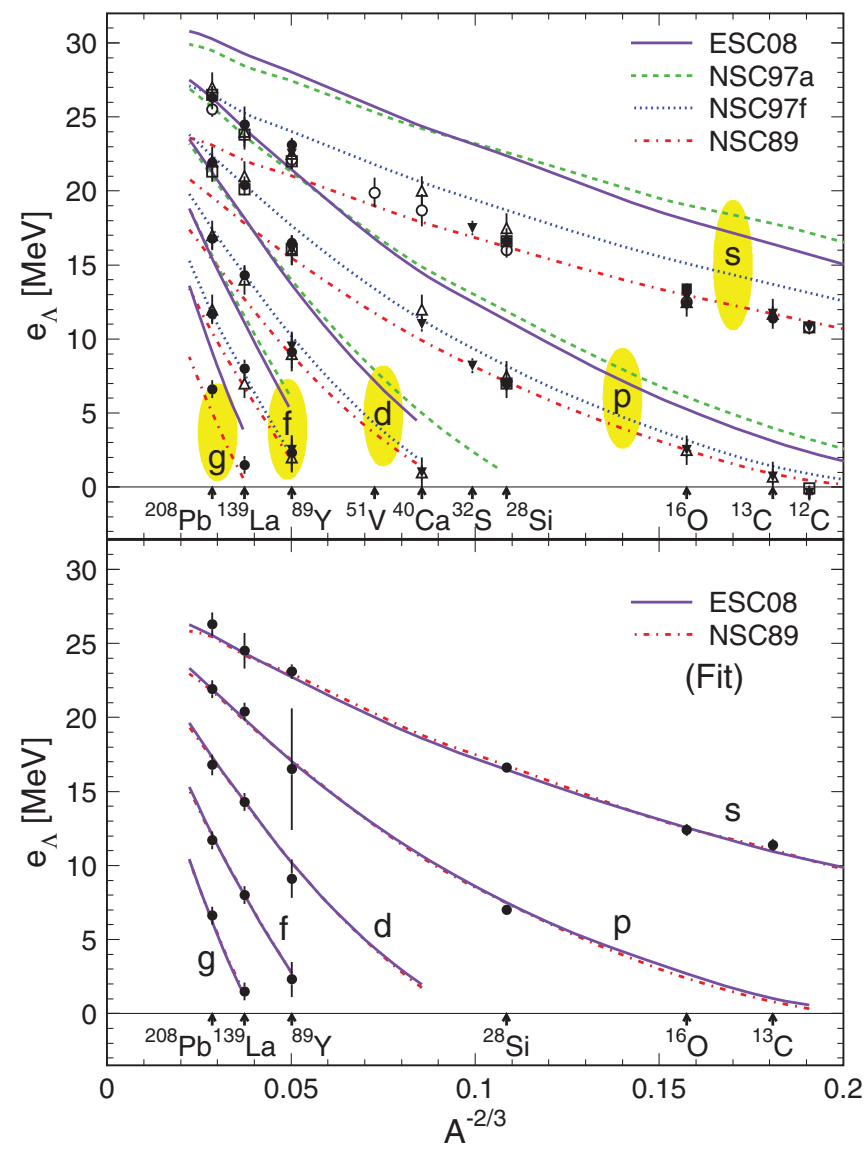

FIG. 3. (Color online) $\Lambda$ s.p. energies in different single- $\Lambda$ hypernuclei. Markers indicate experimental data [27,28] and lines the theoretical predictions obtained with different $N Y$ potentials (top panel) and with the fitted versions of Sec. VI (bottom panel).

comprises the complete set of $S=-2 Y Y$ interactions, and it is therefore of interest to examine its predictions for double- $\Lambda$ hypernuclei, while the NSC89 model without $Y Y$ interaction serves for a reference calculation.

We list in Table $\mathrm{V}$ the bond energy

$$
\Delta B_{\Lambda \Lambda}=2 E\left({ }_{\Lambda}^{A-1} Z\right)-E\left({ }_{\Lambda \Lambda}^{A} Z\right)-E\left({ }^{A-2} Z\right),
$$

TABLE V. Bond energies, Eq. (28), of several double- $\Lambda$ hypernuclei, obtained with different potentials.

\begin{tabular}{lcccc}
\hline \hline & \multicolumn{4}{c}{$\Delta B_{\Lambda \Lambda}[\mathrm{MeV}]$} \\
\cline { 2 - 5 } & NSC89 & NSC97a & NSC97f & ESC08 \\
${ }_{\Lambda \Lambda}^{A} Z$ & -0.23 & +0.10 & -0.25 & -0.57 \\
\hline${ }_{\Lambda \Lambda}^{6} \mathrm{He}$ & -0.34 & +0.37 & -0.35 & -0.41 \\
${ }_{\Lambda \Lambda}^{10} \mathrm{Be}$ & -0.41 & +0.32 & -0.47 & -0.53 \\
${ }_{\Lambda \Lambda}^{14} \mathrm{C}$ & -0.41 & +0.32 & -0.41 & -0.37 \\
${ }_{\Lambda \Lambda}^{18} \mathrm{O}$ & -0.33 & +0.25 & -0.35 & -0.31 \\
${ }_{\Lambda \Lambda}^{30} \mathrm{Si}$ & -0.31 & +0.19 & -0.32 & -0.23 \\
${ }_{\Lambda \Lambda}^{42} \mathrm{Ca}$ & -0.21 & +0.09 & -0.24 & -0.15 \\
${ }_{\Lambda \Lambda}^{92} \mathrm{Zr}$ & -0.14 & +0.05 & -0.18 & -0.07 \\
${ }_{\Lambda \Lambda}^{142} \mathrm{Ce}$ & -0.12 & +0.01 & -0.15 & -0.09 \\
${ }^{210} \mathrm{~Pb}$ & & & & \\
\hline \hline
\end{tabular}

evaluated for several double- $\Lambda$ hypernuclei with the different potentials. From Figs. 1 (right panel) and 2 (lower panel) one notes that the effective $\Lambda \Lambda$ interaction in nuclear matter is weakly repulsive and consequently the bond energy turns out negative (repulsive) with the ESC08 force. In fact it is very similar to the results obtained with the NSC89 model without any $Y Y$ interaction, which yields also small negative bond energies originating from the momentum dependence of the $\Lambda$ s.p. potential [the $\varepsilon_{4}$ term in Eq. (25)], see Ref. [11]. The NSC97 potentials do contain $Y Y$ forces, but predict also repulsive (NSC97f) or too small (NSC97a) results compared to the recent experimental value for the ${ }_{\Lambda \Lambda}^{6} \mathrm{He}$ nucleus [15], $\Delta B_{\Lambda \Lambda} \approx+0.67 \pm 0.17 \mathrm{MeV}$, indicating a rather weak $\Lambda \Lambda$ attraction.

One should remark that the treatment of the lightest nuclei $\left({ }_{\Lambda \Lambda}^{6} \mathrm{He},{ }_{\Lambda \Lambda}^{10} \mathrm{Be}\right)$ is probably not reliable in the mean-field SHF method, and a cluster [29,30] or shell-model [31] approach should be followed. Also, in view of the very large cancellations observed for the decomposition of the s.p. potentials, and the resulting strong in-medium dependence of the effective $\Lambda \Lambda$ interaction, see Fig. 2 (lower panel), careful fine-tuning of the various components of the $S=-2 Y Y$ interaction will be necessary in order to reliably reproduce the very weak effective interaction observed experimentally. This is certainly a difficult problem for the future, when also reliable data for heavier $\Lambda \Lambda$ hypernuclei will become available.

\section{FIT OF HYPERNUCLEAR DATA}

Our approach so far is not devised to provide perfect reproduction of present hypernuclear data, but rather to assess the consistency of the bare $N Y$ and $Y Y$ potentials with those data. The theoretical method involves necessarily several approximations: The BHF approach of bulk hypernuclear matter is based on the summation of ladder diagrams and neglects in particular higher-order correlations (three-hole line diagrams [32]), hyperonic TBF [33], and relativistic effects [34], for example. Going from bulk matter to finite nuclei involves a local-density approximation, currently neglecting surface effects, spin-orbit forces, etc. Also the SHF approach itself is of course a phenomenological one, involving several approximations [24].

Of all these items, hyperonic TBF $(N N Y, N Y Y, Y Y Y)$ are currently conjectured to be an essential class of corrections that could in particular have important implications at large baryon density and therefore for astrophysical applications [16]. We therefore would like to estimate the required size of these corrections within our current theoretical approach for hypernuclear structure. For this purpose we extend the energy density functional, Eq. (25), with terms that mimic the effects of hyperonic TBF. Obviously the TBF depend on the chosen baryon-baryon potential, ESC08 or NSC89. In fact in the former case it is clear from Fig. 3 (top panel) that the effect of TBF on the effective $N \Lambda$ interaction must be overall repulsive, while attractive in the latter case.

A natural choice for the leading corrections to the energy density caused by hyperonic TBF is an expression of the 
functional form

$$
\varepsilon_{N \Lambda}^{\mathrm{TBF}}\left(\rho_{N}, \rho_{\Lambda}\right)=\tilde{\varepsilon}_{1} \rho_{N} \rho_{N} \rho_{\Lambda}+\tilde{\varepsilon}_{2} \rho_{N} \rho_{\Lambda} \rho_{\Lambda}+\tilde{\varepsilon}_{3} \rho_{\Lambda} \rho_{\Lambda} \rho_{\Lambda},
$$

where the $\tilde{\varepsilon}_{i}$ are fit parameters, to be adjusted by a better reproduction of the hypernuclear spectra, Fig. 3, for example.

Note that terms of the functional form $\sim \tilde{\varepsilon}_{1}, \tilde{\varepsilon}_{3}$ are already contained in the original functional Eq. (25) $\left(\sim \varepsilon_{2}, \varepsilon_{8}\right.$; also the $\tilde{\varepsilon}_{2}$ term corresponds roughly to the $\varepsilon_{5}$ one), but they (and higher-order terms) stem from the nonlinear density dependence of the s.p. potentials caused solely by two-body forces within the BHF method. The last contribution in Eq. (29) does not depend on $\rho_{N}$ and thus the $\Lambda$ s.p. energies in single- $\Lambda$ hypernuclei are not very sensitive to it. We therefore reserve it for a future fit of heavy double- $\Lambda$ hypernuclei, once available, whereas with the two parameters $\tilde{\varepsilon}_{1}, \tilde{\varepsilon}_{2}$ alone an excellent reproduction of single- $\Lambda$ spectra can be achieved.

For definiteness, in order to adjust these parameters we choose the experimental data on the single- $\Lambda s, p, d, f, g$ levels of the nuclei ${ }_{\Lambda}^{13} \mathrm{C},{ }_{\Lambda}^{16} \mathrm{O},{ }_{\Lambda}^{28} \mathrm{Si},{ }_{\Lambda}^{89} \mathrm{Y},{ }_{\Lambda}^{139} \mathrm{La},{ }_{\Lambda}^{208} \mathrm{~Pb}$, published in Ref. [28]. Currently the experimental accuracy is generally not much better than about $1 \mathrm{MeV}$ for heavy nuclei, and therefore a precision fit, eventually involving a bigger data set, is not feasible for the moment. This will hopefully improve in the nearer future. In practice rather convincing results can be obtained that are plotted in Fig. 3 (lower panel) for the NSC89 and ESC08 forces together with the data set of the selected nuclei. In fact the results for both potentials are nearly indistinguishable. The corresponding fit parameters $\tilde{\varepsilon}_{i}$ are listed in Table IV. As expected, the leading correction $\sim \tilde{\varepsilon}_{1}$ compared to the original term $\sim \varepsilon_{2}$ is about $10 \%$ and attractive for the NSC89 model, and about $20 \%$ and repulsive for the ESC08. The rms deviation of the theoretical and experimental s.p. energies is about $0.4 \mathrm{MeV}$ and thus within present experimental accuracy.

\section{CONCLUSIONS}

We confronted the predictions of the new ESC08 NY $+Y Y$ model with current experimental data on single- and double$\Lambda$ hypernuclei. A two-step BHF + SHF theoretical approach that allows to treat the complicated coupled-channel structure was employed and we discussed the results obtained for bulk hypernuclear matter and various hypernuclei. In particular we found some overbinding of single- $\Lambda$ hypernuclei and a weakly repulsive $\Lambda \Lambda$ bond energy with the ESC08 model.

In the future, more precise experimental data on heavy single- and double- $\Lambda$ hypernuclei are needed in order to (a) fine-tune the bare $N Y, Y Y$ interactions and (b) better estimate the empirical corrections due to three-body forces and other many-body effects. The presented theoretical framework is ready for this task. A high-precision energy density functional could then be used to make predictions for other hypernuclei and also provide hints for the hypernuclear matter calculations relevant for astrophysical applications.

\section{ACKNOWLEDGMENTS}

We would like to acknowledge valuable discussions with E. Hiyama, N. Itagaki, T. Motoba, M. M. Nagels, A. Ohnishi, and Y. Yamamoto.
[1] A. Gal, Int. J. Mod. Phys. E 19, 2301 (2010); E. Botta, T. Bressani, and G. Garbarino, Eur. Phys. J. A 48, 41 (2012); H. Tamura, Prog. Theor. Exp. Phys. 02B012 (2012).

[2] M. M. Nagels, T. A. Rijken, and J. J. de Swart, Phys. Rev. D 15, 2547 (1977); 20, 1633 (1979).

[3] P. M. M. Maessen, T. A. Rijken, and J. J. de Swart, Phys. Rev. C 40, 2226 (1989).

[4] A. Reuber, K. Holinde, and J. Speth, Nucl. Phys. A 570, 543 (1994); J. Haidenbauer and Ulf-G. Meissner, Phys. Rev. C 72, 044005 (2005); J. Haidenbauer, Few-Body Syst. 54, 85 (2013).

[5] T. Fujita, Y. Fujiwara, C. Nakamoto, and Y. Suzuki, Prog. Theor. Phys. 100, 931 (1998).

[6] K. Tominaga et al., Nucl. Phys. A 642, 483 (1998).

[7] T. A. Rijken, V. G. J. Stoks, and Y. Yamamoto, Phys. Rev. C 59, 21 (1999); V. G. J. Stoks and T. A. Rijken, ibid. 59, 3009 (1999).

[8] T. Rijken, M. Nagels, and Y. Yamamoto, Nucl. Phys. A 835, 160 (2010); Y. Yamamoto, E. Hiyama, and T. Rijken, ibid. 835, 350 (2010); T. Rijken, M. Nagels, and Y. Yamamoto, Prog. Theor. Phys. Suppl. 185, 14 (2010); Few-Body Syst. 54, 801 (2013).

[9] H.-J. Schulze, M. Baldo, U. Lombardo, J. Cugnon, and A. Lejeune, Phys. Rev. C 57, 704 (1998).

[10] I. Vidaña, A. Polls, A. Ramos, M. Hjorth-Jensen, and V. G. J. Stoks, Phys. Rev. C 61, 025802 (2000).
[11] J. Cugnon, A. Lejeune, and H.-J. Schulze, Phys. Rev. C 62 , 064308 (2000); I. Vidaña, A. Polls, A. Ramos, and H.-J. Schulze, ibid. 64, 044301 (2001); H.-J. Schulze, Nucl. Phys. A 835, 19 (2010)

[12] X. R. Zhou, H.-J. Schulze, H. Sagawa, C. X. Wu, and E. G. Zhao, Phys. Rev. C 76, 034312 (2007); H.-J. Schulze, M. Thi Win, K. Hagino, and H. Sagawa, Prog. Theor. Phys. 123, 569 (2010).

[13] X. R. Zhou, A. Polls, H.-J. Schulze, and I. Vidaña, Phys. Rev. C 78, 054306 (2008).

[14] V. G. J. Stoks, R. A. M. Klomp, M. C. M. Rentmeester, and J. J. de Swart, Phys. Rev. C 48, 792 (1993).

[15] H. Takahashi et al., Phys. Rev. Lett. 87, 212502 (2001); S. Aoki et al. (KEK E176 Collaboration), Nucl. Phys. A 828, 191 (2009); K. Nakazawa et al., ibid. 835, 207 (2010); K. Nakazawa and H. Takahashi, Prog. Theor. Phys. Suppl. 185, 335 (2010).

[16] M. Baldo, G. F. Burgio, and H.-J. Schulze, Phys. Rev. C 61, 055801 (2000); H.-J. Schulze, A. Polls, A. Ramos, and I. Vidaña, ibid. 73, 058801 (2006); Z. H. Li and H.-J. Schulze, ibid. 78, 028801 (2008); H.-J. Schulze and T. Rijken, ibid. 84, 035801 (2011).

[17] R. B. Wiringa, V. G. J. Stoks, and R. Schiavilla, Phys. Rev. C 51, 38 (1995). 
[18] P. Grangé, A. Lejeune, M. Martzolff, and J.-F. Mathiot, Phys. Rev. C 40, 1040 (1989); W. Zuo, A. Lejeune, U. Lombardo, and J.-F. Mathiot, Nucl. Phys. A 706, 418 (2002); Eur. Phys. J. A 14, 469 (2002); Z. H. Li, U. Lombardo, H.-J. Schulze, and W. Zuo, Phys. Rev. C 77, 034316 (2008).

[19] K. A. Brueckner and J. L. Gammel, Phys. Rev. 109, 1023 (1958); J.-P. Jeukenne, A. Lejeune, and C. Mahaux, Phys. Rep. 25, 83 (1976); M. Baldo, Nuclear Methods and the Nuclear Equation of State (World Scientific, Singapore, 1999).

[20] H. Noumi et al., Phys. Rev. Lett. 89, 072301 (2002); P. K. Saha et al., Phys. Rev. C 70, 044613 (2004); M. Kohno, Y. Fujiwara, Y. Watanabe, K. Ogata, and M. Kawai, ibid. 74, 064613 (2006); M. Agnello et al. (Finuda Collaboration), Nucl. Phys. A 835, 398 (2010).

[21] P. Khaustov et al. (AGS E885 Collaboration), Phys. Rev. C 61, 054603 (2000)

[22] D. J. Millener, C. B. Dover, and A. Gal, Phys. Rev. C 38, 2700 (1988).

[23] D. Vautherin and D. M. Brink, Phys. Rev. C 5, 626 (1972); D. Vautherin, ibid. 7, 296 (1973).

[24] M. Bender, P.-H. Heenen, and P.-G. Reinhard, Rev. Mod. Phys. 75, 121 (2003); J. R. Stone and P.-G. Reinhard, Prog. Part. Nucl. Phys. 58, 587 (2007); J. Erler, P. Klüpfel, and P.-G. Reinhard, J. Phys. G 38, 033101 (2011).

[25] H. Kohri et al., Phys. Rev. C 65, 034607 (2002); T. Motoba, D. E. Lanskoy, D. J. Millener, and Y. Yamamoto, Nucl. Phys. A 804, 99 (2008); F. Cusanno et al., Phys. Rev. Lett. 103, 202501 (2009); T. Motoba, P. Bydžovský, M. Sotona, and K. Itonaga, Prog. Theor. Phys. Suppl. 185, 224 (2010).

[26] E. Chabanat, P. Bonche, P. Haensel, J. Meyer, and R. Schaeffer, Nucl. Phys. A 627, 710 (1997); 635, 231 (1998); 643, 441 (1998).
[27] R. E. Chrien et al., Nucl. Phys. A 478, 705c (1988); P. H. Pile et al., Phys. Rev. Lett. 66, 2585 (1991); S. Ajimura et al., Nucl. Phys. A 585, 173c (1995); T. Hasegawa et al., Phys. Rev. C 53, 1210 (1996); H. Hotchi et al., ibid. 64, 044302 (2001); D. H. Davis, Nucl. Phys. A 754, 3 (2005); M. Agnello et al. (Finuda Collaboration), Phys. Lett. B 698, 219 (2011).

[28] O. Hashimoto and H. Tamura, Prog. Part. Nucl. Phys. 57, 564 (2006).

[29] A. R. Bodmer and Q. N. Usmani, Nucl. Phys. A 468, 653 (1987); Q. N. Usmani, A. R. Bodmer, and B. Sharma, Phys. Rev. C 70, 061001(R) (2004); A. A. Usmani and Z. Hasan, ibid. 74, 034320 (2006).

[30] E. Hiyama, M. Kamimura, Y. Yamamoto, and T. Motoba, Phys. Rev. Lett. 104, 212502 (2010); E. Hiyama, M. Kamimura, Y. Yamamoto, T. Motoba, and T. A. Rijken, Prog. Theor. Phys. Suppl. 185, 152 (2010); E. Hiyama, Few-Body Syst. 53, 189 (2012)

[31] A. Gal and D. J. Millener, Phys. Lett. B 701, 342 (2011).

[32] H. Q. Song, M. Baldo, G. Giansiracusa, and U. Lombardo, Phys. Rev. Lett. 81, 1584 (1998); M. Baldo, A. Fiasconaro, H. Q. Song, G. Giansiracusa, and U. Lombardo, Phys. Rev. C 65, 017303 (2001).

[33] A. A. Usmani, Phys. Rev. C 52, 1773 (1995); A. A. Usmani and F. C. Khanna, J. Phys. G 35, 025105 (2008); T. Takatsuka, S. Nishizaki, and Y. Yamamoto, Eur. Phys. J. A 13, 213 (2002); I. Vidaña, D. Logoteta, C. Providência, A. Polls, and I. Bombaci, Europhys. Lett. 94, 11002 (2011).

[34] R. Brockmann and W. Weise, Nucl. Phys. A 355, 365 (1981); G. E. Walker, ibid. 450, 287 (1986). 